\title{
Optimal Multivalued Shattering
}

\author{
Zoltán Füredi ${ }^{12} \quad$ Attila Sali ${ }^{34}$
}

\footnotetext{
${ }^{1}$ Department of Mathematics University of Illinois at Urbana-Champaign 1409 W. Green Street Urbana, Illinois 61801-2975, USA. E-mail: z-furedi@illinois.edu and Alfréd Rényi Institute of Mathematics Hungarian Academy of Sciences Budapest, P.O.Box 127 H-1364 Hungary.

${ }^{2}$ Research supported in part by the Hungarian National Science Foundation OTKA, by the National Science Foundation under grant NFS DMS 09-01276, and by the European Research Council Advanced Investigators Grant 267195.

${ }^{3}$ Alfréd Rényi Instiute of Mathematics Hungarian Academy of Sciences Budapest, P.O.Box 127 H-1364 Hungary. E-mail: sali@renyi.hu

${ }^{4}$ Partially supported by Hungarian National Science Foundation OTKA grant No. NK 78439.

This copy was printed on May 5, 2022, multishatter $0907 . t e x$
}

Version as of September 7, 2011. 


\begin{abstract}
We have found the most general extension of the celebrated Sauer, Perles and Shelah, Vapnik and Chervonenkis result from 0-1 sequences to $k$-ary codes still giving a polynomial bound.

Let $\mathcal{C} \subseteq\{0,1, \ldots, k-1\}^{n}$ be a $k$-ary code of length $n$. For a subset of coordinates $S \subset\{1,2, \ldots, n\}$ the projection of $\mathcal{C}$ to $S$ is denoted by $\left.\mathcal{C}\right|_{S}$. We say that $\mathcal{C}(i, j)$-shatters $S$ if $\left.\mathcal{C}\right|_{S}$ contains all the $2^{|S|}$ distinct vectors (codewords) with coordinates $i$ and $j$. Suppose that $\mathcal{C}$ does not $(i, j)$-shatter any coordinate set of size $s_{i, j} \geq 1$ for every $1 \leq i<j \leq q$ and let $p=\sum\left(s_{i, j}-1\right)$. Using a natural induction we prove that

$$
|\mathcal{C}| \leq O\left(n^{p}\right)
$$

for any given $p$ as $n \rightarrow \infty$ and give a construction showing that this exponent is the best possible.

Several open problems are mentioned.
\end{abstract}

Keywords: shattering, VC-dimension, forbidden configurations 


\section{Introduction}

Let $[n]$ denote the set $\{1,2, \ldots, n\}$ while let $(k)$ denote $\{0,1, \ldots, k-1\}$ and for any set $S$, let $2^{S}$ denote the family of all $2^{|S|}$ subsets of $S$ and let $\left(\begin{array}{c}S \\ k\end{array}\right)$ denote all $\left(\begin{array}{c}|S| \\ k\end{array}\right)$ subsets of $S$ of size $k$. Consider a family $\mathcal{F}$ of subsets of $[n]$. We say that $\mathcal{F}$ shatters $S$ if

$$
\{E \cap S: E \in \mathcal{F}\}=2^{S}
$$

The following result has a variety of applications including learning theory and applied probability.

Theorem 1 [Sauer[12], Perles, Shelah[13], Vapnik, Chervonenkis[15] Let $\mathcal{F}$ be a family of subsets of $[n]$ with no shattered set of size $s$. Then

$$
|\mathcal{F}| \leq\left(\begin{array}{c}
n \\
s-1
\end{array}\right)+\left(\begin{array}{c}
n \\
s-2
\end{array}\right)+\cdots+\left(\begin{array}{l}
n \\
0
\end{array}\right)
$$

and this bound is the best possible.

Karpovsky and Milman [10] and independently Steele [14] gave a multivalued generalization of the result above. Let $\mathcal{C} \subseteq(k)^{n}$ be a set of codewords (vectors). A codeword $\mathbf{c}$ can also be viewed as a function from $[n]$ to $(k)$. The code $\mathcal{C}$ is said to shatter $S \subseteq[n]$ if

$$
\left\{\left.\mathbf{c}\right|_{S}: \mathbf{c} \in \mathcal{C}\right\}=(k)^{S},
$$

the set of all functions from $S$ to $(k)$.

Theorem 2 [Karpovsky and Milman [10] and independently Steele [14] (see also Frankl [5], Alon [1], Anstee [2])] Let $1 \leq s \leq n$ be an integer and let $\mathcal{C} \subseteq(k)^{n}$ be a set of codewords with no shattered set of size s. Then

$$
|\mathcal{C}| \leq \sum_{i=0}^{s-1}(k-1)^{n-i}\left(\begin{array}{l}
n \\
i
\end{array}\right) .
$$

An important difference between the bounds is that (1) is polynomial in $n$ (for fixed $s$ ), but (2) is exponential. The same phenomenon happens when uniform set systems are considered. The uniform version of Theorem 1 was proven by Frankl and Pach [6] (for a strengthening and algebraic connections see Anstee et.al. [4]). 
Theorem 3 [Frankl and Pach [6] Let $n, d, s$ be positive integers such that $d \leq n$ and $s \leq n / 2$. Let $\mathcal{F} \subseteq\left(\begin{array}{c}{[n]} \\ d\end{array}\right)$ be a d-uniform set system that does not shatter an s-element set, then

$$
|\mathcal{F}| \leq\left(\begin{array}{c}
n \\
s-1
\end{array}\right)
$$

Recently, Hegedüs and Rónyai [9] gave two multivalued generalizations.

Theorem 4 [Hegedüs and Rónyai [9]] Let $0 \leq d \leq(k-1) n$ and $s-1 \leq n / 2$. Let $\mathcal{C} \subseteq(k)^{n}$ be a code with no shattered set of size $s$ and suppose that $\sum_{i=1}^{n} c_{i}=d$ for every $\mathbf{c} \in \mathcal{C}$. Then

$$
|\mathcal{C}| \leq \sum_{i=0}^{s-1}(k-1)^{n-i}\left(\left(\begin{array}{c}
n \\
i
\end{array}\right)-\left(\begin{array}{c}
n \\
i-1
\end{array}\right)\right)
$$

Note that this bound is exponential in $n$.

Theorem 5 [Hegedüs and Rónyai [9]] Let $0 \leq d \leq n$ and $0 \leq d+s \leq n+1$. Let $\mathcal{C} \subseteq(k)^{n}$ be a code with no shattered set of size $s$ and suppose that $\left|\left\{i \in[n]: c_{i} \neq 0\right\}\right|=d$ for every $\mathbf{c} \in \mathcal{C}$. Then

$$
|\mathcal{C}| \leq\left(\begin{array}{c}
n \\
s-1
\end{array}\right) \sum_{i=0}^{d}(k-2)^{i}\left(\begin{array}{c}
n-s+1 \\
i
\end{array}\right)
$$

One cannot expect an exponential bound here since the total number of codewords with support of size $d$ is polynomial.

A code $\mathcal{C}$ and the corresponding matrix $\mathcal{M}$ formed by the codewords are called reverse-free if $\mathcal{M}$ does not have a submatrix of the form $\left(\begin{array}{cc}a & b \\ b & a\end{array}\right)$ for any distinct $a$ and $b$. How large a reverse-free code $\mathcal{C} \subset(k)^{n}$ can be? It was proved in [7] that

$$
\max |\mathcal{C}|=\Theta\left(n\left(\begin{array}{c}
k \\
2
\end{array}\right)\right)
$$

This can lead to the following version of multivalued shattering. Let $\mathcal{C} \subseteq(k)^{n}$ be a set of codewords. $\mathcal{C}(i, j)$-shatters $S \subseteq[n]$ if $\left.\mathcal{C}\right|_{S}$ contains all $2^{|S|}$ functions from $S$ to $\{i, j\}$. Let $k \geq 2$ be a fixed integer, $\vec{s}=\left(s_{0,1}, s_{0,2}, \ldots s_{k-2, k-1}\right)$ be a positive integer vector of length $\left(\begin{array}{l}k \\ 2\end{array}\right)$ whose entries are indexed by ordered pairs $(i, j)$ with $0 \leq i<j \leq k-1$.

The main result of the present paper is the following theorem. 
Theorem 6 Suppose that $\mathcal{C} \subset(k)^{n}$ does not $(i, j)$-shatter any coordinate set of size $s_{i, j} \geq 1$ for every $0 \leq i<j \leq k-1$. Then

$$
|\mathcal{C}| \leq \sum_{0 \leq \alpha_{i, j} \leq s_{i, j}-1}\left(\begin{array}{c}
n \\
\alpha_{0,1}, \alpha_{0,2}, \ldots, \alpha_{k-2, k-1}, n-\sum_{0 \leq i<j \leq k-1} \alpha_{i, j}
\end{array}\right)=O\left(n^{p}\right)
$$

where the sum is taken for all possible choices of $\alpha_{i, j}$ 's and $p=\sum_{0 \leq i<j \leq k-1}\left(s_{i, j}-1\right)$.

On the other hand, when $p$ is fixed and $n \rightarrow \infty$ then there exist codes $\mathcal{C} \subset(k)^{n}$ such that they do not $(i, j)$-shatter any coordinate set of size $s_{i, j} \geq 1$ for every $0 \leq i<j \leq k-1$ and

$$
|\mathcal{C}|=\Omega\left(n^{p}\right)
$$

In other words, if forb $(n, \vec{s})$ denotes the maximum number of codewords of a code $\mathcal{C}$ of length $n$ over the alphabet $(k)$ such that $\mathcal{C}$ does not $(i, j)$-shatter any coordinate set of size $s_{i, j}$ then

$$
\operatorname{forb}(n, \vec{s})=\Theta\left(n^{p}\right)
$$

\section{A hierarchy of Vapnik-Chervonenkis type dimensions}

The VC-dimension of a set system $\mathcal{F} \subseteq 2^{[n]}$ is the maximum $d$ that $\mathcal{F}$ shatters a set of size $d$. Theorem 1 bounds the size of a set system whose VC-dimension is less than $s$. Vapnik and Chervonenkis used it for bounds on the sample size necessary to obtain uniformly good empirical estimates for the expectations of all random variables of a given class. Since then it has found applications in learning theory, such as concepts with bounded VC-dimensions are effectively learnable.

Theorem 2 allows the definition of another dimension, KM-dimension of codes (systems of multisets) as follows. The KM-dimension of $\mathcal{C} \subseteq(k)^{n}$ is the maximum $d$ that $\mathcal{C}$ shatters a set of size $d$. Theorem 2 gives a bound on the size of a code of KM-dimension less than $s$. However, this bound is exponential function of $n$.

Haussler and Long [8] introduced other generalizations of VC-dimension, motivated by statistical applications. The G-dimension of $\mathcal{C} \subseteq(k)^{n}$ is the maximum $d$ that there exists a vector $\vec{y}=$ $\left(y_{1}, y_{2}, \ldots y_{d}\right) \in(k)^{d}$ and a subset $D=\left\{i_{1}, i_{2}, \ldots i_{d}\right\} \subseteq[n]$ such that for all subsets $I \subseteq D$ there exists $\mathbf{c}=\left(c_{1}, c_{2}, \ldots c_{n}\right) \in \mathcal{C}$ such that $c_{i_{j}}=y_{j}$ for $i_{j} \in I$ and $c_{i_{t}} \neq y_{t}$ for $i_{t} \notin I$. 
The P-dimension of $\mathcal{C}$ is the maximum $d$ that there exists a vector $\vec{y}$ and a subset $|D|=d$ of $[n]$ such that for all subsets $I \subseteq D$ there exists $\mathbf{c} \in \mathcal{C}$ such that $c_{i_{j}} \geq y_{j}$ for $i_{j} \in I$ and $c_{i_{t}}<y_{t}$ for $i_{t} \notin I$.

The GP-dimension of $\mathcal{C}$ is the maximum $d$ that there exists a vector $\vec{y}$ and a subset $|D|=d$ of $[n]$ such that for all subsets $I \subseteq D$ there exists $\mathbf{c} \in \mathcal{C}$ such that $c_{i_{j}}=y_{j}$ for $i_{j} \in I$ and $c_{i_{t}}<y_{t}$ for $i_{t} \notin I$.

Finally, the $\mathrm{N}$-dimension (or Natarajan-dimension [11]) of $\mathcal{C}$ is the maximum $d$ that there exist vectors $\vec{y}$ and $\vec{z}$ with $z_{i}<y_{i}: i=1,2, \ldots d$ and a subset $|D|=d$ of $[n]$ such that for all subsets $I \subseteq D$ there exists $\mathbf{c} \in \mathcal{C}$ such that $c_{i_{j}}=y_{j}$ for $i_{j} \in I$ and $c_{i_{t}}=z_{t}$ for $i_{t} \notin I$.

It is easy to see that each of the above dimensions coincide with the VC-dimension in the case of $k=1$. We also have

$$
\operatorname{dim}_{\mathrm{KM}}(\mathcal{C}) \leq \operatorname{dim}_{\mathrm{N}}(\mathcal{C}) \leq \operatorname{dim}_{\mathrm{GP}}(\mathcal{C}) \leq\left\{\begin{array}{l}
\operatorname{dim}_{\mathrm{G}}(\mathcal{C}) \\
\operatorname{dim}_{\mathrm{P}}(\mathcal{C})
\end{array}\right.
$$

The concept of $(i, j)$-shattering allows us to define a new dimension which is between KM-dimension and N-dimension.

The bi-dimension of $\mathcal{C} \subseteq(k)^{n}$ is the maximum $d$ that there exist $i<j \in(k)$ and and a set $D \subseteq[n]$ of size $d$ that $\mathcal{C}(i, j)$-shatters $D$. If a set $D$ is KM-shattered by $\mathcal{C}$, then $\left.\mathcal{C}\right|_{D}$ is the set of all functions from $D$ to $(k)$, in particular it contains all functions from $D$ to $\{i, j\}$ for any pair $i<j \in(k)$, so $D$ is $(i, j)$-shattered by $\mathcal{C}$. This shows

$$
\operatorname{dim}_{\mathrm{KM}}(\mathcal{C}) \leq \operatorname{dim}_{\mathrm{bi}}(\mathcal{C})
$$

On the other hand, if $D$ is $(i, j)$-shattered by $\mathcal{C}$, then $D$ satisfies the condition of N-dimension with vectors $\vec{z}=(i, i, \ldots, i)$ and $\vec{y}=(j, j, \ldots, j)$, so the $\mathrm{N}$-dimension of $\mathcal{C}$ is at least as large as its bi-dimension.

Let $\mathcal{M}_{\mathrm{X}}(n, s)$ denote the maximum size of a code of length $n$ and $\mathrm{X}$-dimension not exceeding $s$ $(\mathrm{X} \in\{\mathrm{KM}, \mathrm{bi}, \mathrm{N}, \mathrm{GP}, \mathrm{G}, \mathrm{P}\})$. Then (6) and the observations above imply

$$
\left.\begin{array}{l}
\mathcal{M}_{\mathrm{G}}(n, s) \\
\mathcal{M}_{\mathrm{P}}(n, s)
\end{array}\right\} \leq \mathcal{M}_{\mathrm{GP}}(n, s) \leq \mathcal{M}_{\mathrm{N}}(n, s) \leq \mathcal{M}_{\mathrm{bi}}(n, s) \leq \mathcal{M}_{\mathrm{KM}}(n, s)
$$


In fact, Haussler and Long [8] proved that

$$
\begin{gathered}
\mathcal{M}_{\mathrm{G}}(n, s)=\mathcal{M}_{\mathrm{P}}(n, s)=\mathcal{M}_{\mathrm{GP}}(n, s)=\sum_{0 \leq i \leq s}\left(\begin{array}{l}
n \\
i
\end{array}\right)(k-1)^{i} . \\
\mathcal{M}_{\mathrm{N}}(n, s) \leq \sum_{0 \leq i \leq s}\left(\begin{array}{l}
n \\
i
\end{array}\right)\left(\begin{array}{l}
k \\
2
\end{array}\right)^{i}
\end{gathered}
$$

These bounds are polynomial in $n$. Theorem 6 implies that $\mathcal{M}_{\mathrm{bi}}(n, s)$ is polynomial, as well, since $\mathcal{M}_{\mathrm{bi}}(n, s)=\operatorname{forb}(n, \vec{s})$ for the vector $\vec{s}$ whose coordinates are all $s+1$. However, $\mathcal{M}_{\mathrm{KM}}(n, s)$ is exponential according to Theorem 2, An extremal property of bi-dimension is that it is the weakest restriction that still results in polynomial bound. Indeed, if there is a pair of symbols $i, j$ such that there is no restriction involving only that pair, then one can select all codewords $\mathcal{C}=\{i, j\}^{n}$ so that $\mathcal{C}$ does not violate any restrictions yet it is of exponential size.

\section{Proofs}

In this section we give two versions of the proof of the upper bound in Theorem 6 . The lower bound (5) follows from Proposition 7 .

Branching proof. Let $\mathcal{C} \subset(k)^{n}$ be a code avoiding an $(i, j)$-shattered set of size $s_{i, j}$ for all $0 \leq i<j \leq k-1$. The following branching process will be applied to $\mathcal{C}$ successively $n$ times.

Let $\mathcal{B}$ be a set of codewords of length $t \geq 1$ over alphabet $(k)$. Let $\mathcal{B}_{0}$ denote the set of suffices of length $t-1$ of codewords in $\mathcal{B}$. Note, that if $t=1$, then $\mathcal{B}_{0}$ has one element, the empty string. If a codeword $\mathbf{b} \in \mathcal{B}_{0}$ appears with more than one first coordinate in $\mathcal{B}$, say with $i_{1}<i_{2}<\ldots<i_{w}$, then $\mathbf{b}$ will be put into the $(w-1)$ sets $\mathcal{B}_{i_{1}, i_{2}}, \mathcal{B}_{i_{1}, i_{3}}, \ldots, \mathcal{B}_{i_{1}, i_{w}}$. We get

$$
|\mathcal{B}|=\left|\mathcal{B}_{0}\right|+\sum_{0 \leq i<j \leq k-1}\left|\mathcal{B}_{i, j}\right| .
$$

$\mathcal{B}_{i, j}$ is said to be obtained by $(i, j)$-branching at step $t$ from $\mathcal{B}$.

Thus, the process starts with $\mathcal{B}=\mathcal{C}$ and $t=n$, and continues with $t=n-1, n-2, \ldots, 1$. At step $t$ every set of codewords obtained at step $t+1$ is branched. At the end, there are $|\mathcal{C}|$ singleton 

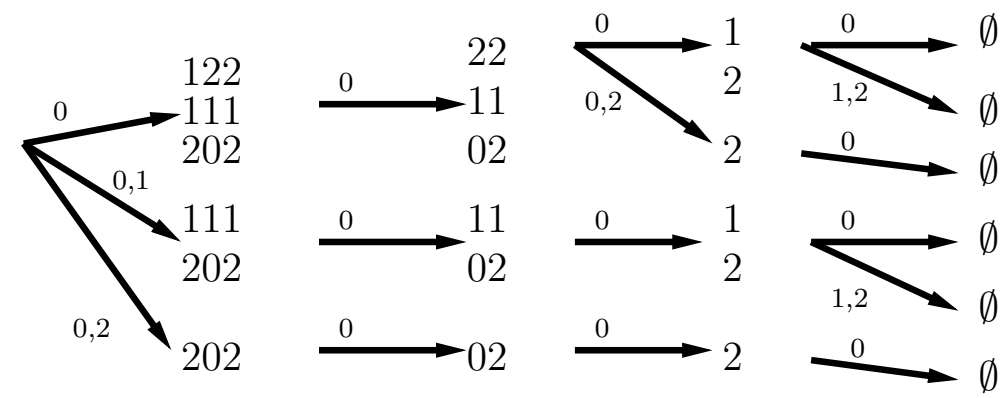

Figure 1: Branching example

sets each containing the empty string. For an example see Figure 1. Every singleton set is a result of a series of branchings, say $\alpha_{i, j}(i, j)$-branchings for $0 \leq i<j \leq k-1$. If $\alpha_{i, j} \geq s_{i, j}$ for some pair $i, j$, and these branchings occur at steps $t_{1}, t_{2}, \ldots t_{\alpha_{i, j}}$, then $\mathcal{C}(i, j)$-shatters the set $\left\{t_{1}, t_{2}, \ldots t_{\alpha_{i, j}}\right\}$ that contradicts the assumptions. The maximum possible number of singleton sets with $\alpha_{i, j}(i, j)$ branchings is equal to the number of $n$-permutations of $\alpha_{i, j}$ objects of type $(i, j)$ for $0 \leq i<j \leq k-1$ and $n-\sum_{0 \leq i<j \leq k-1} \alpha_{i, j}$ objects of "no branching" type, which is exactly the multinomial coefficient

$$
\left(\begin{array}{c}
n \\
\alpha_{0,1}, \alpha_{0,2}, \ldots, \alpha_{k-2, k-1}, n-\sum_{0 \leq i<j \leq k-1} \alpha_{i, j}
\end{array}\right) .
$$

This provides the upper bound (4).

Induction proof. Let $\mathcal{C}_{i, j}^{i} \subseteq \mathcal{C}$ consist of those codewords $\mathbf{c}$ that $c_{n}=i$ and there exists a codeword $\mathbf{c}^{\prime} \in \mathcal{C}$ that only differs from $\mathbf{c}$ in the last coordinate and $c_{n}^{\prime}=j . \mathcal{C}_{i, j}^{j} \subseteq \mathcal{C}$ is defined similarly. If $s_{i, j}=1$, then both $\mathcal{C}_{i, j}^{i}$ and $\mathcal{C}_{i, j}^{j}$ are empty. Otherwise, let $\vec{s}_{i, j}$ be the vector obtained from $\vec{s}$ by decreasing the $(i, j)^{\text {th }}$ coordinate by one. Then obviously $\left|\mathcal{C}_{i, j}^{i}\right|=\left|\mathcal{C}_{i, j}^{j}\right| \leq$ forb $\left(n-1, \vec{s}_{i, j}\right)$. Let $\left.\mathcal{C}\right|_{[n-1]}=\left\{\left.\mathbf{c}\right|_{[n-1]}: \mathbf{c} \in \mathcal{C}\right\}$ be the set of length $n-1$ prefixes of codewords in $\mathcal{C}$. Clearly, $\left.\mathcal{C}\right|_{[n-1]} \leq$ forb $(n-1, \vec{s})$. On the other hand,

$$
|\mathcal{C}| \leq|\mathcal{C}|_{[n-1]}\left|+\sum_{0 \leq i<j \leq k-1}\right| \mathcal{C}_{i, j}^{j} \mid
$$

In order to prove (4) using induction we have to give upper bound for forb $(1, \vec{s})$. In this case $i$ and $j$ both can be codewords in $\mathcal{C}$ iff $s_{i, j}>1$. Let $G_{\vec{s}}=((k), E)$ be the graph on vertex set $(k)$ be defined 
by $\{i, j\} \in E \Longleftrightarrow s_{i, j}>1$. Then

$$
\operatorname{forb}(1, \vec{s})=\omega\left(G_{\vec{s}}\right)
$$

It is an easy exercise that the right hand side of (4) is an upper bound for this clique number in case of $n=1$. The bound in (4) follows from (7) and (8) using induction and the well-known recurrence for the multinomial coefficients.

\section{Forbidden configurations}

Another generalization or sharpening of Theorem 1 considers forbidden configurations. We say a $(0,1)$-matrix is simple if there are no repeated rows. Given a $(0,1)$-matrix $F$, we say a matrix $A$ has $F$ as a configuration denoted $F \in A$, if there is a submatrix of $A$ which is a row and column permutation of $F$. Let $|A|$ denote the number of rows of matrix $A$. We define

$$
\text { forb }(n, F)=\max \{|A|: A \text { is a simple 0-1 matrix without configuration } F \text { of } n \text { columns }\} \text {. }
$$

A simple (0,1)-matrix $A$ naturally corresponds to a set system $\mathcal{F}_{A}$ taking the rows as characteristic vectors of subsets of $[n] . \mathcal{F}_{A}$ shatters an $s$-set iff $A$ has the $2^{s} \times s$ configuration of all distinct rows of size $s$.

The concept of forbidden configurations can be extended for matrices of entries from $(k)$. A $(k)$-matrix is simple if there are no repeated rows. Given a $(k)$-matrix $F$, we say a matrix $A$ has $F$ as a configuration denoted $F \in A$, if there is a submatrix of $A$ which is a row and column permutation

of $F$. Theorem 2 gives upper bound on $m$ for an $m \times n$ simple $(k)$-matrix that does not have the $k^{s} \times s$ configuration of all distinct rows of size $s$.

Definition (9) of forb $(n, F)$ can be applied to $(k)$-matrices, as well. However, if polynomial upper bounds are desired, then more than one configurations must be forbidden simultaneously. Let $\mathcal{F}=\left\{F_{1}, F_{2}, \ldots, F_{t}\right\}$ be a collection of (not necessarily simple) $(k)$-matrices. Let

forb $(n, k, \mathcal{F})=\max \{m: A$ is $m \times n$ simple $(k)$-matrix and has no configuration $F \in \mathcal{F}\}$. 
In [7] it was proved that

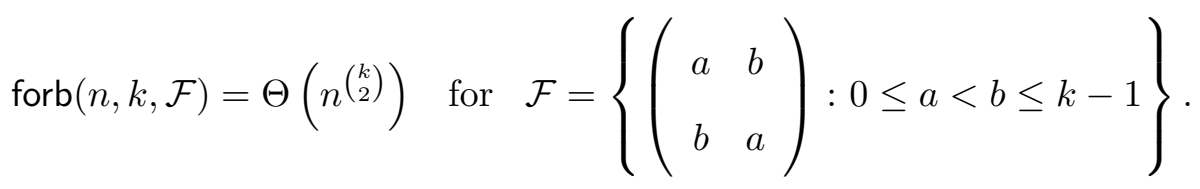

Theorem 6 can also be reformulated in this language. Let $F$ be a $(0,1)$-matrix, then $F(i, j)$ denotes the $(i, j)$-matrix obtained from $F$ replacing 0 's by $i$ 's and 1's by $j$ 's. Let $K_{s}$ denote the $2^{s} \times s$ $(0,1)$-matrix of all distinct rows of size $s$. Theorem 6 gives bounds for forb $(n, k, \mathcal{F})$ where $\mathcal{F}=$ $\left\{K_{s_{i, j}}(i, j): 0 \leq i<j \leq k-1\right\}$. Here we prove a lower bound.

Proposition 7 Let $F^{i, j}: 0 \leq i<j \leq k-1$ be simple $(0,1)$-matrices such that none of them contains a constant column. Then

$$
\operatorname{forb}\left(n, k,\left\{F^{i, j}(i, j): 0 \leq i<j \leq k-1\right\}\right) \geq \prod_{0 \leq i<j \leq k-1} \text { forb }\left(\frac{n}{\left(\begin{array}{c}
k \\
2
\end{array}\right)}, F^{i, j}\right)
$$

Proof: We apply the product construction introduced in [3]. Let $A^{i, j}$ be a simple $(0,1)$-matrix with $\frac{n}{\left(\begin{array}{c}k \\ 2\end{array}\right)}$ columns and forb $\left(\frac{n}{\left(\begin{array}{c}k \\ 2\end{array}\right)}, F^{i, j}\right)$ rows without configuration $F^{i, j}$. Let

$$
A=A^{0,1} \times A^{0,2} \times \ldots \times A^{k-2, k-1}
$$

be the matrix with $n$ columns and $\left|A^{0,1}\right| \cdot\left|A^{0,2}\right| \cdot \ldots \cdot\left|A^{k-2, k-1}\right|$ rows obtained by choosing one row from each of the matrices and putting them side by side in every possible way. We claim that this product matrix $A$ avoids all configurations $F^{i, j}: 0 \leq i<j \leq k-1$. Indeed, since each column of $F^{i, j}$ contains both symbols $i$ and $j$, columns of a configuration $F^{i, j}$ should come from columns of $A^{i, j}$ in the product. Suppose $F^{i, j}$ has $p$ columns. Since $F^{i, j}$ is simple and $A^{i, j}$ does not have configuration $F^{i, j}$, for each $p$-tuple of columns of $A^{i, j}$ there must be a row of $F^{i, j}$ that is missing on those columns. This will be missing in the product matrix, as well.

Lower bound $(5)$ follows by taking $F^{i, j}=K_{s_{i, j}}: 0 \leq i<j \leq k-1$ and applying Theorem 1 . 


\section{Open problems}

There are more questions than answers known in connection with $(i, j)$-shattering. The principal problem is that Theorem 6 does not give sharp bounds, in contrast with Theorem 1 and Theorem 2. We can give an exact bound only if most of the $s_{i, j}$ 's are ones.

Proposition 8 Assume that $s_{i, j}=1$ if $i<j<k-1$. Then

$$
\operatorname{forb}(n, k, \vec{s})=\max _{\sum_{i=0}^{k-2} n_{i}=n} \prod_{i=0}^{k-2}\left(\left(\begin{array}{c}
n_{i} \\
s_{i, k-1}-1
\end{array}\right)+\left(\begin{array}{c}
n_{i} \\
s_{i, k-1}-2
\end{array}\right)+\ldots+\left(\begin{array}{c}
n_{i} \\
0
\end{array}\right)\right)
$$

Proof: Suppose that $A$ is a $(k)$-matrix without configurations $K_{s_{i, j}} . s_{i, j}=1$ means that symbols $i$ and $j$ cannot occur in the same column of $A$. Thus columns of $A$ can be partitioned into $k-1$ parts, part $C_{i}$ containing only symbols $i$ and $k-1$ for $0 \leq i<k-1$. The number of different projections onto column set $C_{i}$ is $\left(\begin{array}{c}n_{i} \\ s_{i, k-1}-1\end{array}\right)+\left(\begin{array}{c}n_{i} \\ s_{i, k-1}-2\end{array}\right)+\ldots+\left(\begin{array}{c}n_{i} \\ 0\end{array}\right)$ for $n_{i}=\left|C_{i}\right|$ by Theorem 1. Thus the maximum number of different rows of $A$ is at most $\prod_{i=0}^{k-2}\left(\left(\begin{array}{c}n_{i} \\ s_{i, k-1}-1\end{array}\right)+\left(\begin{array}{c}n_{i} \\ s_{i, k-1}-2\end{array}\right)+\ldots+\left(\begin{array}{c}n_{i} \\ 0\end{array}\right)\right)$. On the other hand the product construction 10 provides a matching lower bound.

It would be interesting to find exact bounds for other special cases, as well.

Another question whether containing no constant column or simplicity of the forbidden configurations is necessary condition in Proposition 7. Also, Proposition 7 and Theorem 6 give asymptotically tight bounds if forb $\left(n, F^{i, j}\right)=\Theta\left(n^{s_{i, j}-1}\right)$ where $s_{i, j}$ is the number of columns of $F^{i, j}$. The question is that does Proposition 7 give the correct order of magnitude of forb $(n, k, \mathcal{F})$ for other lists $\mathcal{F}$ of forbidden configurations?

Since VC-dimension has many of applications in statistics, computer science and combinatorics, it seems likely that bi-dimension can be applied there, too. 


\section{References}

[1] N. Alon, On the density of sets of vectors, Discrete Math. 46 (1983), 199-202.

[2] R. P. Anstee, A forbidden configuration theorem of Alon, J. Combin. Th. A 47 (1988), 16-27.

[3] R. P. Anstee, J. R. Griggs, A. Sali, Small forbidden configurations, Graphs and Combinatorics 13 (1997), 97-118.

[4] R. P. Anstee, L. Rónyai and A. Sali, Shattering news, Graphs and Combin. 18 (2002), 59-73.

[5] P. Frankl, On the trace of finite sets, Journal of Combinatorial Theory, Ser. A 34 (1983), 41-45.

[6] P. Frankl and J. Pach, On disjointly representable sets, Combinatorica 4 (1984), 39-45.

[7] Z. Füredi, I. Kantor, A. Monti, and B. Sinaimeri, On reverse-free codes and permutations, SIAM J. Discrete Math. 24 (2010), 964-978.

[8] D. Haussler and P. M. Long, A generalization of Sauer's lemma, Journal of Combinatorial Theory, Ser. A 71 (1995), 219-240.

[9] G. Hegedüs and L. Rónyai, Multivalued generalizations of the Frankl-Pach Theorem, to appear in J. of Algebra and its Applications http://arxiv.org/abs/1008.4660v2

[10] M. G. Karpovsky and V.D. Milman, Coordinate density of vectors, Discrete Math. 24 (1978), $177-184$.

[11] B. K. Natarajan, On learning sets and functions, Machine Learning 4 (1989), 67-97.

[12] N. Sauer, On the density of families of sets, J. Combin. Th. A 13 (1972), 145-147.

[13] S. Shelah, A combinatorial problem: Stability and order for models and theories in infinitary language, Pacific J. Math. 41 (1972), 247-261.

[14] J. M. Steele, Existence of submatrices with all possible columns, J. Combin. Th. A 24 (1978), $84-88$.

[15] V. N. Vapnik and A. Ya. Chervonenkis, On the uniform convergence of relative frequencies of events to their probabilities, Theory Prob. Applics. 16 (1971), 264-280. 